\title{
LncRNA HCG11 suppresses the progression of breast cancer through cooperating with miR-330- 3p/FOX01 axis
}

Xiaotao Zhu ( $\sim$ zxt-304@163.com )

Jinhua central hospital

Fan Wang

Jinhua central hospital

Mingzheng Wang

Jinhua central hospital

Lin LV

Jinhua central hospital

Dan He

Jinhua central hospital

Chen Fan

Jinhua central hospital

\section{Research Article}

Keywords: Breast cancer, IncRNA HCG11, miR-330-3p, FOXO1

Posted Date: March 1st, 2022

DOI: https://doi.org/10.21203/rs.3.rs-1345638/v1

License: (1) This work is licensed under a Creative Commons Attribution 4.0 International License.

Read Full License 


\section{Abstract}

Background: Breast cancer is the most common cancer among women and the second most common cancer among newly diagnosed cancers worldwide. At present, long non-coding RNAs (IncRNAs) are widely reported to be involved in the occurrence and development of multiple cancers. As a newly discovered long non-coding RNA, the function of HLA complex group 11 (HCG11) remain uncertain in breast cancer. This article aims to examine the effect of HCG11 in breast cancer.

Methods: RT-PCR was performed to detect the mRNA level of HCG11 in both breast cancer tissues and cell lines. HCG11 overexpression virus and siHCG11 were constructed and transfected to the breast cancer cell line MCF-7. The effect of HCG11 were evaluated by CCK-8 assay, colony formation assay, wound scratch assay, and cell invasion assay. Then we found and determined small RNA that may interact with HCG11 by bioinformatics analysis and luciferase report analysis. Next, we further researched the role of miR-330-3p in HCG11-mediated breast cancer by CCK-8 assay, colony formation assay, wound scratch assay, and cell invasion assay. At last, the above experimental methods and nude mouse experiments were used to confirm the role of HCG11/miR-330-3p/ FOXO1 in breast cancer process.

Results: The results showed that the mRNA level of HCG11 was low-expressed in breast cancer tissues and cell lines. HCG11 overexpression inhibited cell proliferation, invasion and migration in MCF-7 cell line. Moreover, HCG11 could target miR-330-3p/FOXO1 and further suppressed the progression of breast cancer.

Conclusion: In summary, IncRNA HCG11 suppressed the progression of breast cancer through cooperating with miR-330-3p/FOXO1 axis

\section{Background}

Breast cancer has become the first malignant tumor among women worldwide, with the leading morbidity and mortality rate, seriously endangering women's lives and health $[1,2]$. The global statistics from the American Cancer Society showed that estimately 268600 newly diagnosed invasive breast cancers and approximately 41760 deaths of breast cancer patients occurred in women worldwide in 2019, which accounted for a third of all new diagnosed cancer among women [3].

Long-chain non-coding RNAs (IncRNAs) are new types of RNA molecule that don't encode protein [4]. They participate in regulating gene expression at the level of epigenetics, gene transcription and posttranscription, and can interact with proteins and nucleic acids to participate in regulation of various physiological and pathological processes [5-7]. Studies have shown that IncRNAs are abnormally expressed in a variety of cancer cells and play important roles in the initiation, development, invasion and metastasis of cancers [8-10]. The genesis, development and metastasis of breast cancer are regulated by multiple genes and factors. Different IncRNA molecules have different regulatory functions and mechanisms for breast cancer [11]. HLA complex 11 (HCG11) as a newly discovered IncRNA, it has been 
regarded as a tumor suppressor for prostate cancer [12]. And some studies reported that HCG11 inhibits tumor cell apoptosis in liver cancer research [13]. However, at present, the effect of HCG11 on the biological behavior of breast cancer and its regulatory mechanism are still unclear. Thus, s elucidate the role of HCG11 in the progression of breast cancer.

Recent studies have shown that IncRNA can also be used as a competitive endogenous RNA (ceRNA) to interact with miRNA, and they are involved in tumorigenesis and development $[14,15]$. MicroRNA is a type of small non-coding RNA with about 18-22 nucleotides, which regulates gene expression and affects cell development, proliferation, differentiation, and apoptosis $[16,17]$. In the study, the potential target miR330-3p of HCG11 was investigated. The underlying mechanism in the role of HCG11 through cooperating with miR-330-3p/FOXO1 axis in breast cancer was studied. The purpose of the article is to elaborate the role of HCG11/miR-330-3p/FOXO1 axis in breast cancer.

\section{Materials And Methods Sample collection}

There are altogether 78 pairs of breast cancer tissue samples and adjacent healthy tissue samples which were collected from breast cancer patients in Jinhua central hospital. We have collected informed consents from all patients and the research was supported by the ethics committee of Jinhua central hospital. All samples were collected in liquid nitrogen and frozen in $-80^{\circ} \mathrm{C}$ refrigerator for later

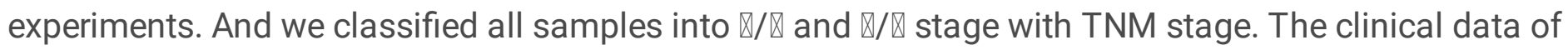
patients were as follows: male 8 , female 70 . About TNM stage, $\varangle / \bigotimes$ stage $34, \varangle / \varangle$ stage 44 .

\section{Cell culture}

Normal breast epithelial cell line MCF-10A and four different breast cancer cell lines MCF-7, T47D, SKBR3, MDA-MB-231 were purchased from Shanghai Cell Bank of the Chinese Academy of Sciences (Shanghai, China). All the cells were cultured in an environment of Dulbecco's modified Eagle's medium (DMEM) (Gibco, Gaithersburg, MD, USA) medium with $10 \%$ fetal bovine serum (FBS) (Sigma, St. Louis, MO, USA), containing $1 \%$ penicillin/streptomycin in $37{ }^{\circ} \mathrm{C}$ incubator with $5 \% \mathrm{CO}_{2}$.

\section{Cell transfection}

Small interfering RNA against HCG11 (siHCG11) and the corresponding control siRNA (siNC), overexpression vector pcDNA3.1-HCG11 and the corresponding empty vector (NC) were all constructed and synthesized by QIAGEN (Valencia, CA, USA). The miR-330-3p mimic, miR-330-3p inhibitor and their corresponding mimic control or inhibitor control were synthesized by GenePharma (Shanghai, China). They were transfected into MCF-7 cells with lipofectamine 2000 reagent (Invitrogen, \#11668019) for later experiments. RT-PCR was determined to detect the transfection efficiency.

\section{Quantitative real-time polymerase chain reaction (RT-PCR)}


Total RNA was extracted from the breast cancer tissues and adjacent healthy tissues, normal breast epithelial cell line MCF-10A and different breast cancer cell lines including MCF-7. Following the manufacturer's instruction, RNA was collected by using RNAiso Plus reagent (Takara, Code. No. \#9109). Complementary DNA (cDNA) was reverse-transcribed in a total of $10 \mu$ l reaction system with the reagent PrimeScript $^{\text {TM }}$ RT Master Mix (Perfect Real Time) (Takara, Code. No. RR036) (500 ng total RNA, $2 \mu$ l Mix, added to $10 \mu \mathrm{ddH}_{2} \mathrm{O}$ ). Then PCR was performed with the reagent kit (Roche, Code. No. 04913914001) in a $10 \mu$ reaction system, consisting of $5 \mu$ l FastStart Universal SYBR Green Master, $2 \mu$ l cDNA, $1 \mu$ primer and $2 \mu \mathrm{ldd} \mathrm{H}_{2} \mathrm{O}$. Then put the plate in the fluorescence PCR machine (ABI 7500) for $2 \mathrm{~h}$, the procedure set as $50^{\circ} \mathrm{C}$ for $2 \mathrm{~min}, 95^{\circ} \mathrm{C}$ for $10 \mathrm{~min}$, then $95^{\circ} \mathrm{C}$ for $20 \mathrm{~s}, 65^{\circ} \mathrm{C}$ for $20 \mathrm{~s}$ and $72^{\circ} \mathrm{C}$ for $30 \mathrm{~s}$ for amplification, in a total of 40 cycles. Glyceraldehyde-3-phosphate dehydrogenase (GAPDH) RNA was the internal reference of the experiment. The primers used were as follows: HCG11 sense, forward primer: 5'AGGAGTGGTTGCATTTGGGA-3' and reverse primer: 5'-CCCACCACGCAGTGAATAGT-3'; GAPDH sense, forward primer: 5'-GTCACCTTCACCGTTCCAGTTTT-3' and reverse primer: 5'CTTAGTTGCGTTACACCCTTTCTT-3'; miR-330-3p sense, forward primer: 5'-CAACTGCCTCTCTGGGCCTG3 ' and reverse primer: 5'-CTGCAGAGAGGCAGCGCTG-3'; FOXO1 sense, forward primer: 5'-

TCGTCATAATCTGTCCCTACACA-3'; and reverse primer: 5'-CGGCTTCGGCTCTTAGCAAA-3'.

\section{Western blot}

For protein sample preparation, the protein samples were taken from different treated MCF-7 cells with a mixed lysate consisting of protease inhibitors. Then cell lysates fully lysed $30 \mathrm{~min}$ in ice, centrifuged at $12000 \mathrm{~g}$ for $5 \mathrm{~min}$ at $4^{\circ} \mathrm{C}$. After discarding the precipitate, the supernatant was collected and diluted with $5 \times$ loading buffer in a ratio of $1: 4$, then heated for $5 \mathrm{~min}$ at $100{ }^{\circ} \mathrm{C}$ for fully protein denaturation. For electrophoresis, the samples were electrophoretically separated on $10 \%$ SDS-PAGE at 60V for 30 min and $100 \mathrm{~V}$ for 90 min conventionally, then electrotransferred to polyvinylidene difluoride (PVDF) (Millipore, Cat. No. OPVH00010) membranes. After blocking in western blocking fluid (Beyotime, Cat. No. P0023B) for 2 $\mathrm{h}$ at a shaker. Washing the residual liquid, the membranes were incubated well at $4^{\circ} \mathrm{C}$ for overnight with primary antibody which was pre-diluted with western primary antibody dilution buffer (Beyotime, Cat. No. P0023A). After washing the membranes in $1 \times$ TBST for 3 times, the corresponding secondary antibody conjugated with goat anti-rabbit (Beyotime, Cat. No. A0208) or goat anti-mouse HRP (Beyotime, Cat. No. A0216) in a dilution of 1:1000 with secondary antibody dilution buffer were incubated for $2 \mathrm{~h}$. After washing the membranes for 3 times, detection of proteins was performed with BeyoECL Plus kit (Beyotime, Cat. No. P0018). The primary antibodies used in the article were FOX01 (CST, \#2880, rabbit, 1:1000), GAPDH (CST, \#5174, rabbit, 1:1000).

\section{Cytotoxicity assay}

Cells suspension were evenly cultured in a 96-well plate in a concentration of 5000 cells in $100 \mu$ per well at $37^{\circ} \mathrm{C}$ incubator for $24 \mathrm{~h}$. Then cells were treated and continue cultured for $48 \mathrm{~h}$. For detecting cell viability, every well was added with $10 \mu \mathrm{l}$ CCK-8 solution softly and continued full response at incubator for $2 \mathrm{~h}$. The absorbance of the 96 -well plate in $450 \mathrm{~nm}$ wave length in the microplate reader. The result was recorded in an excel table. 


\section{Colony formation assay}

As a density of $1 \times 10^{3}$ cells per well, we seeded MCF-7 cells to six-well plate and cultured in $37^{\circ} \mathrm{C}$ incubator for 2 weeks. Cells culture were terminated when the colonies were formed, then discarded the supernatant, washed twice with phophate-buffered saline (PBS). The colonies were fixed with $4 \%$ paraformaldehyde for 15 min, stained by crystal violet (Sinopharm Chemical Reagent, Shanghai, China) for 20 min. Recorded the number of colonies by using a microscope.

\section{Wound scratch assay}

MCF-7 cells were seeded in six-well plates at $5 \times 10^{5}$ for $24 \mathrm{~h}$ for $70-80 \%$ confluence. After treated cells, continue cultured cells for $48 \mathrm{~h}$. Then scratch the cell monolayer in a straight line with a sharp pipette tip. For creating scratches of similar size to minimize variations due to width differences, the pipette tip should be perpendicular to the bottom of the well point to the straightedge. After scratch, wash away residual cells gently. And cells were treated with Mitomycin C $(10 \mu \mathrm{g} / \mathrm{ml})($ a proliferation inhibitor) to avoid the effect of cell proliferation. Then cultured cells in incubator for $48 \mathrm{~h}$ and acquired image under a phasecontrast microscope.

\section{Cell invasion assay}

$100 \mu \mathrm{l}$ cell suspension with a density of $5 \times 10^{5}$ was added to transwell chambers with $8-\mu \mathrm{m}$ pore membranes, and 24-well plates were added with $600 \mu \mathrm{l}$ DMEM containing of $20 \% \mathrm{FBS}$, incubated at $37^{\circ} \mathrm{C}$ in $5 \% \mathrm{CO}_{2}$ for $48 \mathrm{~h}$. After culture terminated, taken out transwell chambers, and discard cell medium, fixed with $4 \%$ paraformaldehyde for $30 \mathrm{~min}$. Then transwell chambers were stained with $0.1 \%$ crystal violet for 20 min, and wiped off the upper layer of non-invasioned cells with a cotton swab. Imaged and counted under a microscope.

\section{Luciferase reporter assay}

The HCG11, miR-330-3p, and FOXO1 3'- UTR containing the binding sites were amplified and then inserted into the pmirGLO vector of luciferase expression (Promega, Madison, WI, USA) to obtain wildtype HUCG11/FOXO1 (HCG11-WT / FOXO1-WT) and the same way to generate mutant HCG11/FOXO1 (HCG11-MUT / FOX01-MUT) vectors. Cells were grown in 96-well plates and pmirGLO, HCG11/FOXO1 wild-type vector, HCG11/FOXO1 mutant vector, miR-330-3p mimics and the corresponding empty vector mixed with lipofectamine 2000 (Invitrogen, \#11668019), respectively. And then transfected into MCF-7 cells. After transfection for $48 \mathrm{~h}$, luciferase activity was measured by dual luciferase assay (Promega) and normalized to Renilla luciferase activity.

\section{Statistical analysis}

The data were obtained from at least three independent experiments and were shown as the mean \pm SD. The difference of two groups were analyzed by Student's t test. Multiple groups comparisons were compared by one-way analysis of variance (ANOVA), followed by Bonferroni's post hoc test. Comparison of normal breast epithelial cell line and different breast cancer cells, was analyzed by ANOVA, followed by 
Dunnett's post hoc test. P value $<0.05$ was considered statistically significant. The data were analyzed by Graphpad Prism 8 (GraphPad Software, Inc.).

\section{Results}

\section{HCG11 was downregulated in breast cancer tissues and cells}

The expression of HCG11 was detected in different breast cancer tissues and cells by performing RT-PCR assay. HCG11 was discovered to be obviously downregulated in breast cancer tissues of patients, compared with paracancerous tissues (Fig. 1A). To further elucidate the phenomenon, we detected the mRNA level of HCG11 in different stages of breast cancer tissues. We divided the stages of breast cancer

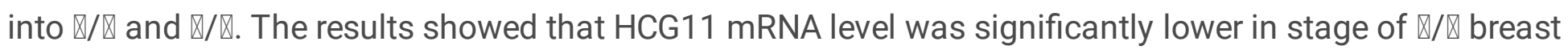

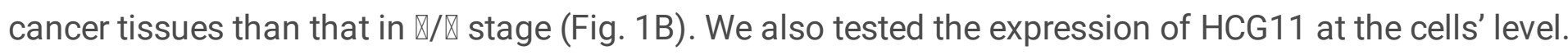
RT-PCR assay results revealed that HCG11 expression was decreased in different breast cancer cells, compared with normal breast epithelial cell line MCF-10A (Fig. 1C). The results showed that the level of HCG11 in MCF-7 cell line was the lowest than other breast cancer cell lines, so we chose MCF-7 cells as the model breast cancer cell line.

Overexpression of HCG11 inhibited cell proliferation, migration and invasion in MCF-7 cells, contrary to that of knocking down of HCG11

To evaluate the effect of HCG11 in breast cancer, we constructed overexpression and knockdown HCG11 adenovirus in MCF-7 cells. RT-PCR was performed to examine the efficiency of overexpressing and knocking down of HCG11. The results showed that knocking down and overexpression of HCG11 were both successful, as NC and siNC for the separate reference (Fig. 2A). CCK8 and colony formation assay were both carried out to examine cell proliferation. After transfection for $48 \mathrm{~h}$ and $72 \mathrm{~h}$, CCK8 was performed to detect cell viability. Obviously, cell viability of MCF-7 after overexpression of HCG11 was high effected and lower vitality than NC group, contrast to the siHCG11 group (Fig. 2B). Colony formation assay analysis also illustrated that the numbers of clone in overexpression HCG11 group was significantly reduced compared with the corresponding NC group, contrast to the siHCG11 group (Fig. 2CD). The migration of MCF-7 cells were detected by wound scratch assay. The results showed that the ability of migration in overexpression HCG11 group was worse than NC group, the opposite of siHCG11 group (Fig. 2E-F). Cell invasion assay was used to evaluate the invasion ability of MCF-7 cells. The number of invasion cells in overexpression HCG11 group were much less than NC group, contrasted to siHCG11 group (Fig. 2G-H).

\section{HCG11 could target with miR-330-3p}

To find the miRNA which interacted with HCG11, Starbase software was used to predict target gene. Among the candidate genes predicted by Starbase software, miR-330-3p plays a tumor inhibitory role in 
breast cancer, so we further verify the targeted binding site of miR-330-3p (Fig. 3A). To confirm whether miR-330-3p interact with HCG11 to regulate cell proliferation, migration and invasion in MCF-7 cells, luciferase reporter assay was performed. The results revealed that miR-330-3p mimics can obviously inhibited the luciferase activity of HCG11-WT, however there is no difference in that of HCG11-MUT group (Fig. 3B). RT-PCR assay results showed that the expression of miR-330-3p was significantly upregulated after knocking down HCG11, contrast to overexpression HCG11 (Fig. 3C). Moreover, RT-PCR assay detected the mRNA level of miR-330-3p in breast cancer tissues and paracancerous tissues. Consistently, it is upregulated in breast cancer tissues (Fig. 3D). Then we transfected miR-330-3p mimic and miR-330$3 p$ inhibitor and corresponding control, miR-330-3p control and miR-330-3p inhibitor control to MCF-7 cells. RT-PCR assay verified that transfections were successful (Fig. 4A). CCK8 assay and colony formation assay results showed that miR-330-3p mimic can effectively improve cell viability and the formation of clones, completely opposite to miR-330-3p inhibitor (Fig. 4B-D). Wound scratch assay results indicated that miR-330-3p mimic can improve cell migration compared with mimic control group, opposite to that of miR-330-3p inhibitor (Fig. 4E-F). Cell invasion assay results revealed that miR-330-3p mimic promoted cell invasion compared to mimic control group, opposite to that of miR-330-3p inhibitor (Fig. 4G-H). There are no significant differences in mimic control/inhibitor control and HCG11 + miR-330$3 p$ mimic group in cell proliferation, migration and invasion.

\section{miR-330-3p could target with F0X01}

Through Target 7.2 database analysis, it was predicted that FOXO1 was the downstream candidate gene of miR-330-3p. The target binding site was shown in the Fig. 5A. Through luciferase reporter assay detection, it found that miR-330-3p mimics can obviously inhibited the luciferase activity of HCG11-WT, however there is no difference in that of HCG11-MUT group (Fig. 5B). RT-PCR and Western blot assay were carried out to detect the expression level of FOXO1 in MCF-7 cells. The results showed that mimic inhibited the mRNA and protein level of FOXO1 compared with mimic control group (Fig. 5C, E-F). RT-PCR assay was further detected the expression of FOXO1 in breast cancer tissues and paracancerous tissues, it was high expressed in breast cancer tissues (Fig. 5D). Transfection of miR-330-3p mimic and F0X01 and the corresponding control and vector to MCF-7 cells were carried out. RT-PCR assay results indicated the successful efficiency of transfection (Fig. 6A). CCK8 assay and colony formation assay were performed to evaluate the ability of cell proliferation. Results showed that mimic control + FOXO1 attenuated cell viability and the number of clones, contrasted to mimic + vector (Fig. 6B-D). Wound scratch assay results showed that mimic control + FOXO1 inhibited cell migration than that of mimic + vector (Fig. 6E-F). Cell invasion assay results revealed that mimic control + FOXO1 reduced the ability of cell invasion than that of mimic + vector (Fig. 6G-H).

\section{HCG11 could suppress cell progression of breast cancer through regulating miR-330-3p/F0X01 axis}


To further study the function of HCG11/miR-330-3p/FOXO1 in the progression of breast cancer. We injected MCF-7 cells to nude mouse for $28 \mathrm{~d}$ to monitor the tumor. The results showed that mimic promoted tumor growth. NC group and HCG11 + mimic + FOXO1 had no differences, but significantly bigger than that of HCG11 and FOXO1 group, which were no differences (Fig. 7A). The results demonstrated that HCG11 could suppress cell progression of breast cancer through regulating miR-3303p/FOXO1 axis.

\section{Discussion}

The article is aiming for researching the function of HCG11 in breast cancer through cooperating with miR-330-3p/FOXO1 axis. Our data showed that HCG11 was low expressed in breast cancer tissues and different breast cancer cells. After overexpression HCG11 inhibited cell proliferation, migration and invasion, opposite to that of knocking down HCG11. Moreover, we found that HCG11 targets to miR-330$3 p$, further affecting cell progression. What's more, miR-330-3p was targeted with FOX01. Overall, our studies suggested a possibility of HCG11 suppressed the progression of breast cancer by regulating miR330-3p/FOX01 axis.

Breast cancer is the number one tumor which has extensive diagnosed in women worldwide [18]. LncRNA is abnormally expressed in breast cancer, which has potential value as an indicator of breast cancer diagnosis and prognosis $[19,20]$. Some studies have reported that HCG11 was high-expressed in gastric cancer and can accelerate proliferation and migration in gastric cancer [21]. Other research have found that HCG11 was low-expressed in non-small-cell lung cancer and blocked cell proliferation and accelerated apoptosis in non-small-cell lung cancer [22]. Moreover, it is also proved that abnormally expressed HCG11 is closely related to poor prognosis in breast cancer [11], but the specific mechanism remains unclear. Our results showed that HCG11 expressed lowly in breast cancer tissues and cells. Moreover, overexpression HCG11 in breast cancer cell line MCF-7 inhibited cell proliferation, migration and invasion. And knocking down HCG11 had the adverse results mentioned above. These data prompted that HCG11 may be a potential molecular target for breast cancer treatment.

Recently, more studies have shown that IncRNA can affect the binding of miRNA and its target genes by binding to miRNA sites to regulate the expression of target genes, which is the ceRNAs regulatory network [23]. In addition, IncRNA can interact with miRNA and participate in regulating the biological behavior of tumors [24]. In this paper, starbase software results predicted that miR-330-3p was the target which regulated by HCG11. miR-330-3p has been reported that plays an important role in breast cancer, lung cancer and liver cancer [25-27]. Clinical samples detection showed that miR-330-3p was high-expressed, which was negative correlation with HCG11. RT-PCR assay results showed that the expression of miR330-3p was significantly down-regulated after overexpression HCG11, however the mRNA level of miR330-3p was up-regulated after knocking down HCG11. Furthermore, HCG11 inhibited cell proliferation, migration and invasion through regulating miR-330-3p. 
It is known that FOX01 plays an indispensable role in transcriptional moderator of cell proliferation, so it is regarded as a vital molecule in the growth and development of tumors [28]. In our study, FOXO1 directly targeted miR-330-3p. Luciferase reporter assay results showed that miR-330-3p mimic decreased the luciferase activity of FOXO1, consistent to the RT-PCR and western blot results. The detection of FOXO1 in breast cancer tissues showed that it is positive correlation with HCG11. Moreover, HCG11 can suppressed tumor growth through regulating miR-330-3p/FOX01 axis.

Although there are important discoveries revealed by these results, there are also limitations. First, the relationship between HCG11 and miR-330-3p/FOXO1 in animal model should be studied. Second, HCG11/miR-330-3p/FOXO1 plays role in breast cancer should be investigated in different breast cancer cell lines.

\section{Conclusion}

In summary, these studies proved evidence that HCG11 suppressed the progression of breast cancer through regulating miR-330-3p/FOXO1 axis. It showed that HCG11 may be a potential target, and thus offer a new strategy to treat breast cancer.

\section{Abbreviations}

long non-coding RNAs

IncRNAs)

HLA complex group 11

HCG11

competitive endogenous RNA

ceRNA

\section{Declarations}

\section{Funding}

Not applicable.

\section{Conflicts of Interest}

The authors declare that they have no competing interests.

\section{Data Availability}

The analyzed data sets generated during the study are available from the corresponding author on reasonable request.

\section{Authors' contributions}


$\mathrm{XZ}$ and FW performed the experiments and conducted data analysis. XZ designed the experiment and revised the manuscript. MW and LL wrote the first version of the manuscript and made the figure. $\mathrm{DH}$ and $\mathrm{CF}$ contributed to literature research and animal experiment.

\section{Ethics approval and consent to participate}

All animal experiments were approved by the Animal Investigation Ethics Committee of Jinhua central hospital (Zhejiang, China). The breast cancer tissues were collected from Jinhua central hospital with written informed consent and permission from the Institutional Review Board. All patients provided written informed consent.

\section{Acknowledgement}

Not applicable.

\section{Consent for publication}

Not applicable.

\section{References}

1. Jemal, A., et al., Global cancer statistics. CA Cancer J Clin, 2011. 61(2): p. 69-90.

2. Siegel, R.L., K.D. Miller, and A. Jemal, Cancer Statistics, 2017. CA Cancer J Clin, 2017. 67(1): p. 7-30.

3. Siegel, R.L., K.D. Miller, and A. Jemal, Cancer statistics, 2019. CA Cancer J Clin, 2019. 69(1): p. 7-34.

4. Han, P. and C.P. Chang, Long non-coding RNA and chromatin remodeling. RNA Biol, 2015. 12(10): p. 1094-8.

5. Mercer, T.R., M.E. Dinger, and J.S. Mattick, Long non-coding RNAs: insights into functions. Nat Rev Genet, 2009. 10(3): p. 155-9.

6. Fatica, A. and I. Bozzoni, Long non-coding RNAs: new players in cell differentiation and development. Nat Rev Genet, 2014. 15(1): p. 7-21.

7. Zhang, L., et al., Long non-coding RNA HCG11 suppresses the growth of glioma by cooperating with the miR-4425/MTA3 axis. J Gene Med, 2019. 21(4): p. e3074.

8. Liu, R., et al., Long noncoding RNA signature in predicting metastasis following tamoxifen treatment for ER-positive breast cancer. Pharmacogenomics, 2018.

9. Fan, Y., et al., Long noncoding RNA HOTTIP as an independent prognostic marker in cancer. Clin Chim Acta, 2018. 482: p. 224-230.

10. Leng, X., et al., Long noncoding RNA AFAP1-AS1 is upregulated in NSCLC and associated with lymph node metastasis and poor prognosis. Oncol Lett, 2018. 16(1): p. 727-732.

11. Liu, H., et al., Long non-coding RNAs as prognostic markers in human breast cancer. Oncotarget, 2016. 7(15): p. 20584-96. 
12. Zhang, Y., et al., Downregulation of long non-coding RNA HCG11 predicts a poor prognosis in prostate cancer. Biomed Pharmacother, 2016. 83: p. 936-941.

13. Xu, Y., et al., Modulation of IGF2BP1 by long non-coding RNA HCG11 suppresses apoptosis of hepatocellular carcinoma cells via MAPK signaling transduction. Int J Oncol, 2017. 51(3): p. 791800 .

14. Gao, H., et al., Prognostic signatures for renal cancer as identified by long non-coding and miRNA competing endogenous network analysis. Oncol Rep, 2018. 40(2): p. 959-967.

15. Zhong, Y., et al., Circular RNAs function as ceRNAs to regulate and control human cancer progression. Mol Cancer, 2018. 17(1): p. 79.

16. Kontomanolis, E.N., S. Kalagasidou, and Z. Fasoulakis, MicroRNAs as Potential Serum Biomarkers for Early Detection of Ectopic Pregnancy. Cureus, 2018. 10(3): p. e2344.

17. Emmanuel, K.N., et al., The Impact of microRNAs in Breast Cancer Angiogenesis and Progression. Microrna, 2019. 8(2): p. 101-109.

18. Gao, P., et al., Nogo-B receptor increases the resistance to tamoxifen in estrogen receptor-positive breast cancer cells. Breast Cancer Res, 2018. 20(1): p. 112.

19. Ye, N., et al., Functional roles of long non-coding RNA in human breast cancer. Asian Pac J Cancer Prev, 2014. 15(15): p. 5993-7.

20. Vikram, R., R. Ramachandran, and K.S. Abdul, Functional significance of long non-coding RNAs in breast cancer. Breast Cancer, 2014. 21(5): p. 515-21.

21. Zhang, H., et al., LncRNA HCG11 promotes proliferation and migration in gastric cancer via targeting miR-1276/CTNNB1 and activating Wnt signaling pathway. Cancer Cell Int, 2019. 19: p. 350.

22. Wang, G., et al., LncRNA HCG11 Suppresses Cell Proliferation and Promotes Apoptosis via Sponging miR-224-3p in Non-Small-Cell Lung Cancer Cells. Onco Targets Ther, 2020. 13: p. 6553-6563.

23. Wang, Y., et al., The Emerging Function and Mechanism of ceRNAs in Cancer. Trends Genet, 2016. 32(4): p. 211-224.

24. Salmena, L., et al., A ceRNA hypothesis: the Rosetta Stone of a hidden RNA language? Cell, 2011. 146(3): p. 353-8.

25. Mesci, A., et al., Targeting of CCBE1 by miR-330-3p in human breast cancer promotes metastasis. $\mathrm{Br}$ J Cancer, 2017. 116(10): p. 1350-1357.

26. Shen, L., et al., miR-330-3p promotes lung cancer cells invasion, migration, and metastasis by directly targeting hSOD2b. Biotechnol Appl Biochem, 2019. 66(1): p. 21-32.

27. Jin, Z., et al., miR-330-3p suppresses liver cancer cell migration by targeting MAP2K1. Oncol Lett, 2019. 18(1): p. 314-320.

28. Zhang, B., et al., FOXO1 is a tumor suppressor in cervical cancer. Genet Mol Res, 2015. 14(2): p. 6605-16.

\section{Figures}




\section{Figure 1}

HCG11 was downregulated in breast cancer tissues and cells. (A-B) The expression of HCG11 in breast cancer tissues and in different stages of breast cancer tissues were detected by RT-PCR. (C) The mRNA level of HCG11 in different breast cancer cell lines and normal cell line were evaluated by RT-PCR. ${ }^{* *} \mathrm{P}<0.01$.

\section{Figure 2}

The function of overexpression and knockdown of HCG11 in MCF-7 cells. MCF-7 cell line was transfected with siHCG11 and control siNC, pcDNA3.1-HCG11 and control NC for $48 \mathrm{~h}$. (A) The transfection efficiency was detected by RT-PCR. (B) CCK-8 was used to evaluate cell viability. (C-D) Colony formation assay evaluated the ability of cell proliferation in MCF-7. (E-F) Wound scratch assay evaluated the ability of cell migration in MCF-7. (G-H) Cell invasion assay evaluated the ability of cell invasion in MCF-7. ${ }^{* *} \mathrm{P}<0.01$.

A

\begin{tabular}{|c|c|c|c|c|c|}
\hline miRNA & GenelD & GeneName & GeneType & TargetSite & Alignment \\
\hline
\end{tabular}

B

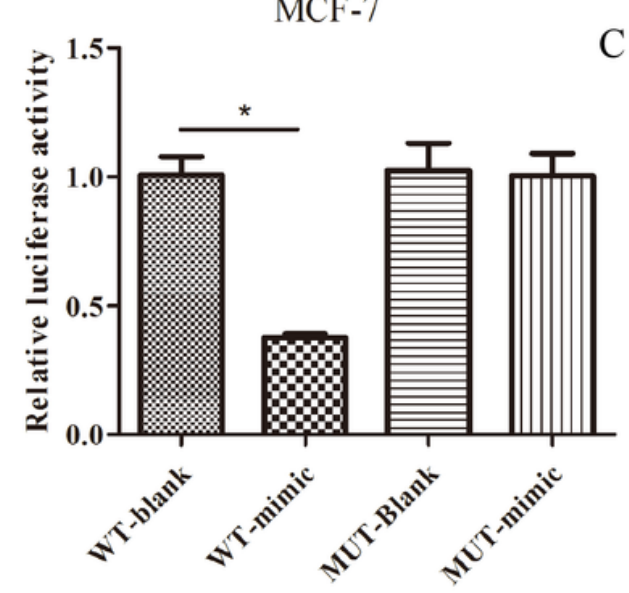

$\mathrm{C}$

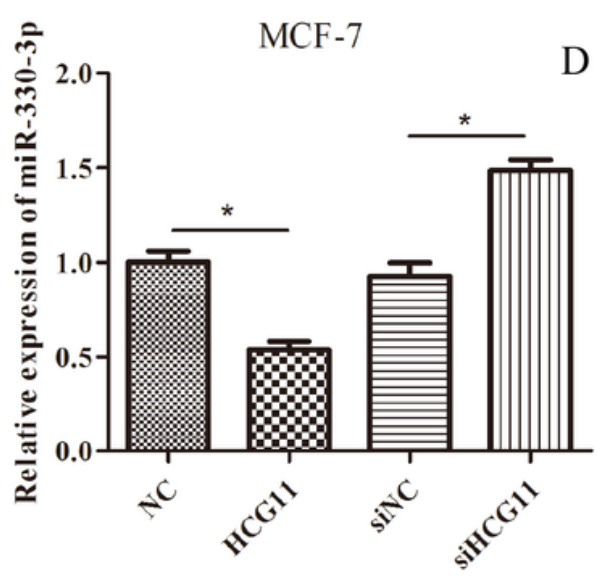

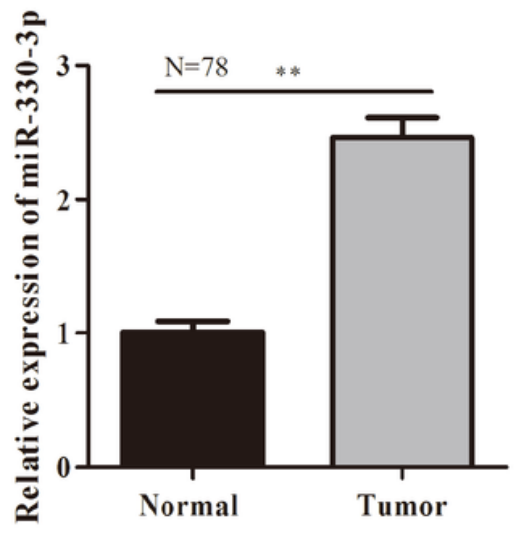

Figure 3

HCG11 could target with miR-330-3p. (A) HCG11 sponge miR-330-3p were predicted by the online prediction software starbase. (B) Luciferase reporter assay was used to detect the luciferase activity. (C) 
RT-PCR detected the expression of miR-330-3p. (D) The miR-330-3p in normal and breast cancer tissues. ${ }^{* *} \mathrm{P}<0.01$.
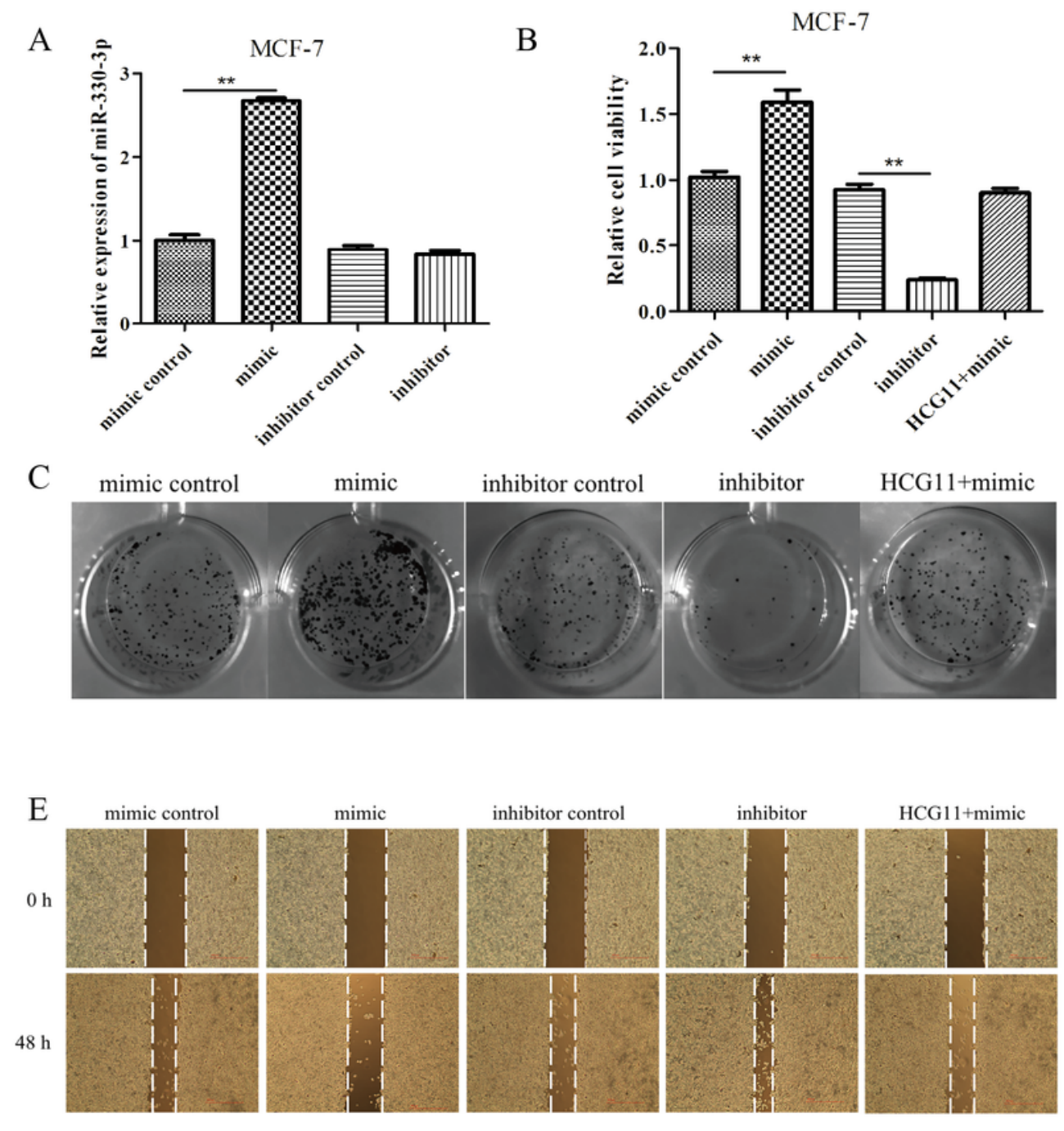

F
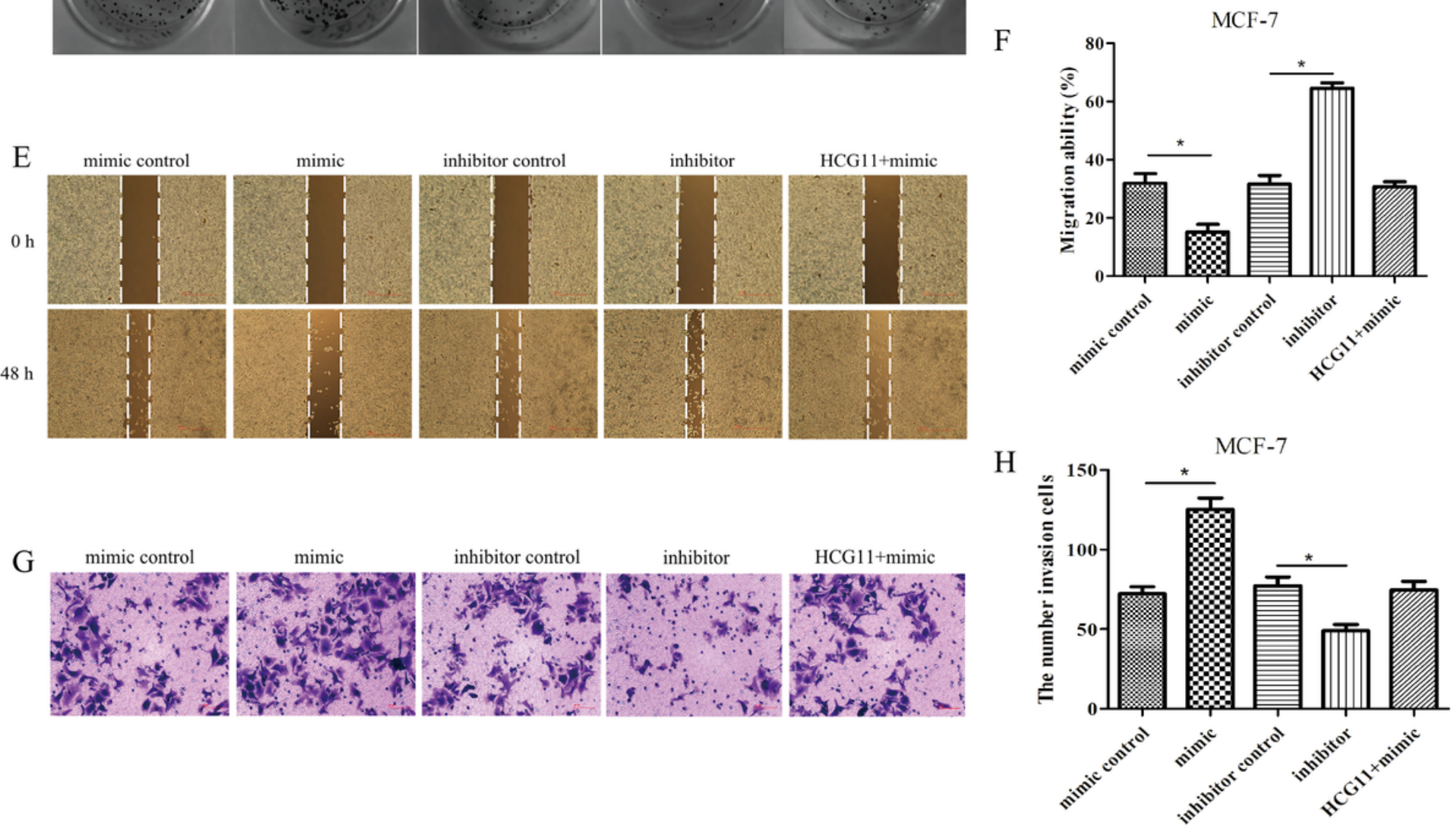

Figure 4

HCG11 affected cell growth by regulating miR-330-3p. MCF-7 cells were transfected with miR-330-3p mimic and mimic control, miR-330-3p inhibitor and inhibitor control, and pcDNA3.1-HCG11 with miR-330$3 p$ mimic. (A) RT-PCR verified the transfection efficiency. (B-D) CCK-8 and colony formation assay 
detected cell proliferation ability. (E-F) Wound scratch assay detected cell migration ability. (G-H) Cell invasion assay detected cell invasion ability. ${ }^{* *} \mathrm{P}<0.01$.

\section{Figure 5}

miR-330-3p could target with FOX01. (A) The binding sites of FOXO1 and miR-330-3p were predicted by the online prediction software TargetScan. (B) Luciferase activity was detected by luciferase reporter assay. (C, E-F) The FOXO1 level was detected by RT-PCR and western blot. (D) Different expression of FOX01 in breast cancer tissues. ${ }^{* *} \mathrm{P}<0.01$.

\section{Figure 6}

miR-330-3p affected cell growth by targeting with FOX01. MCF-7 cells were transfected with miR-330-3p mimic and pcDNA3.1-FOX01, respectively and cotransfection. (A) RT-PCR verified the transfection efficiency. (B-D) CCK-8 and colony formation assay detected cell proliferation ability. (E-F) Wound scratch assay detected cell migration ability. $(\mathrm{G}-\mathrm{H})$ Cell invasion assay detected cell invasion ability. ${ }^{* \star} \mathrm{P}<0.01$. 
A

\title{
HCG11
}

\author{
mimic
}

\section{FOXO1}

\section{HCG11+mimic + FOXO1}
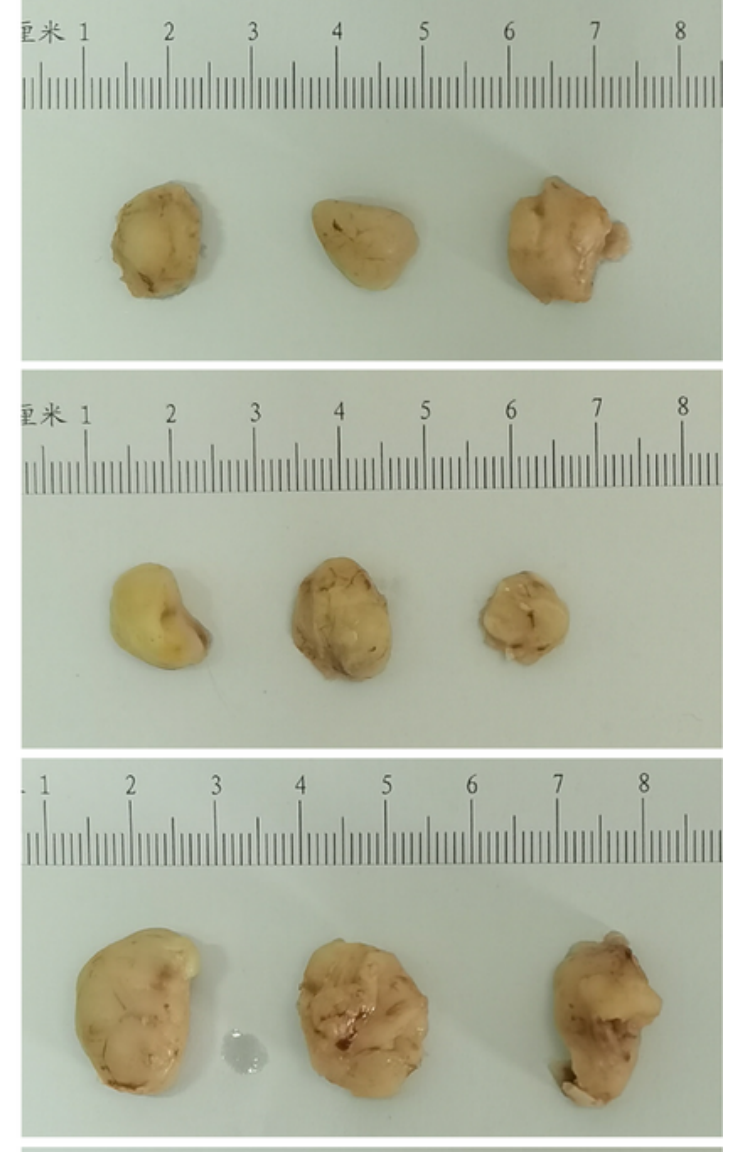